hep-th/9711191

CBPF-NF-052/97

UFES-DF-OP97/2

\title{
On the Non-Renormalization Properties of Gauge Theories with a Chern-Simons Term
}

\author{
Oswaldo M. Del Cima ${ }^{(a),(b), 中, ~ D a n i e l ~ H . T . ~ F r a n c o ~}{ }^{(b)}$,2f, \\ José A. Helayël-Neto ${ }^{(b)}$ and Olivier Piguet ${ }^{(\mathrm{c}), 2, \text {, 色由 }}$ \\ (a) Pontifícia Universidade Católica do Rio de Janeiro (PUC-RIO), \\ Departamento de Física, \\ Rua Marquês de São Vicente, 225 - 22453-900 - Rio de Janeiro - RJ - Brazil. \\ (b) Centro Brasileiro de Pesquisas Físicas (CBPF), \\ Departamento de Teoria de Campos e Partículas (DCP), \\ Rua Dr. Xavier Sigaud 150 - 22290-180 - Rio de Janeiro - RJ - Brazil. \\ (c) Universidade Federal do Espírito Santo (UFES), \\ CCE, Departamento de Física, \\ Campus Universitário de Goiabeiras - 29060-900 - Vitória - ES - Brazil. \\ E-mails: delcima@tph73.tuwien.ac.at,dfranco@cbpfsu1.cat.cbpf.br, \\ helayel@cbpfsu1.cat.cbpf.br,piguet@cce.ufes.br.
}

\footnotetext{
${ }^{1}$ Current address: Institut für Theoretische Physik, Technische Universität Wien, Wiedner Hauptstrasse 8-10 - A-1040 - Vienna - Austria.

${ }^{2}$ Supported by the Conselho Nacional de Desenvolvimento Cientifico e Tecnológico (CNPq).

${ }^{3}$ Supported in part by the Swiss National Science Foundation.

${ }^{4}$ On leave of absence from Département de Physique Théorique, Université de Genève, 24 quai E. Ansermet - CH-1211 - Genève 4 - Switzerland.
} 


\begin{abstract}
Considering three-dimensional Chern-Simons theory, either coupled to matter or with a Yang-Mills term, we show the validity of a trace identity, playing the role of a local form of the Callan-Symanzik equation, in all orders of perturbation theory. From this we deduce the vanishing of the $\beta$-function associated to the Chern-Simons coupling constant and the full finiteness in the case of the Yang-Mills Chern-Simons theory. The main ingredient in the proof of the latter property is the noninvariance of the Chern-Simons form under the gauge transformations. Our results hold for the three-dimensional Chern-Simons model in a general Riemannian manifold.
\end{abstract}

\title{
1 Introduction
}

Topological field theories (see [1] for a general review and references) are a class of gauge model interesting from a physical point of view. In particular, their observables are of topological nature. An important topological model that has received much attention in the last years is the Chern-Simons model in three dimensions [2, 3, 4]. In contrast to the usual Yang-Mills gauge theories, Chern- Simons theories, which include the case of three-dimensional gravity [5, 6], have some remarkable features. Besides their relevance in connection with the possibility of getting nonperturbative results more easily and their relation with the two-dimensional conformal field theories [7], they show very interesting perturbative features such as ultraviolet finiteness [5, 6, 8]. A complete and rigorous proof of the latter property has been given in [9] for $D=1+2$ Chern-Simons theories in the Landau gauge. In the more general case where these models are coupled to matter fields, the Chern-Simons coupling constant keeps unrenormalized, the corresponding $\beta$ function remaining vanishing. This has been rigorously proved in [10 for the Abelian Chern-Simons theory coupled to scalar matter fields. For the nonabelian theory coupled to spinorial matter fields, an argument based on the assumed existence of an invariant regularization has been proposed by the authors of [11].

On the other hand, there exist studies in the literature concerning the Yang-Mills Chern-Simons (YMCS) theory [12, 13, 14, 15]. In particular the finiteness at one loop was detected in refs. [12, 14], where explicit expressions for one-loop radiative corrections can be found. Later on in 15 has been showed that no UV divergences arise up to two-loops in perturbation theory, combining this result with finiteness by power counting at higher loops to argument that theory is finite at any perturbative order. Recently, similar arguments has been used to prove the finiteness for the $N=1$ supersymmetric version of the YMCS theory [16]. A cohomological study of $N=2$ YMCS theory in a nonsupersymmetric gauge has been performed in [17], where a partial proof of the finiteness is given.

Two recent papers [18, 19] have shown the equivalence of the YMCS theory with a pure Cherns-Simons theory, in the classical approximation. One of our results, namely the complete ultraviolet finiteness of the YMCS, although proved in perturbation theory only, indicates that this equivalence could hold for the full quantum theory. Let us finally mention that Ref. [19], appeared after completion of the present paper, beyond 
of expanding the classical results of [18 and arguing in favour of a possible quantum generalization, shows that, at the quantum level, the YMCS theory is finite up to possible field amplitude renormalizations.

Our first purpose in this paper is to give a rigorous proof of the vanishing of the ChernSimons $\beta$-function in the presence of general scalar and spinorial matter, introduced in the most general way compatible with renormalizability. We shall avoid the necessity of invoking a particular regularization procedure, by using the "algebraic" method of renormalization [20], which only relies on general theorems of renormalization theory. We think indeed that, due to the presence of the antisymmetric Levi-Civita tensor, it is difficult to establish an invariant regularization without encountering problems at some or other stage of the argument. Thus, to the contrary of the authors of [11], we shall neither assume an invariant regularization nor the explicit "multiplicative renormalizability" at the level of the Lagrangian which would follow from this assumption.

Our first result generalizes that of [10], the gauge group being now an arbitrary compact Lie group. We exploit the scaling properties of the theory, described by the CallanSymanzik equation, which is the anomalous Ward identity for scale invariance. More precisely, as in [10], we make use of a local form of the Callan-Symanzik equation. But, instead of introducing an external dilatation field as in [10, beyond the external metric or dreibein field, we only consider the latter, considered as a source for the BRS invariant energy-momentum tensor. The conservation of the energy-momentum tensor is expressed through the Ward identity which characterizes the invariance of the theory under the diffeomorphisms. Our "local Callan-Symanzik equation" then is the anomalous Ward identity for the BRS invariant trace of the energy-momentum tensor, the so-called "trace identity". As expected [10, 11], the nonrenormalization of the Chern-Simons coupling, which amounts to the vanishing of the corresponding $\beta$-function, is traced back to the fact that the integrand of the Chern-Simons action is not BRS invariant but transforms as a total derivative.

Of course the $\beta$-functions associated with the other couplings - the self-couplings of the matter fields - do not vanish in general.

Our second result is a purely algebraic proof of the all-order, complete finiteness of the YMCS theory, using the same techniques, but exploiting the superrenormalizability of the model. To our knowledge, up to now, no such algebraic proof had been given. Let us note that, although the proof of finiteness given in [19] confirms our second result in quite an independent way, there are differences which should be noted. First, the result of [19] is more general than ours since it holds for a more general set of theories, which are not necessarely power-counting renormalizable. Second, this result holds only up to possible field amplitude renormalizations. However, restricting ourselves to the powercounting renormalizable case, we are also able to show the complete finiteness, including the nonrenormalization of the field amplitudes, as it is the case for the pure Chern-Simons theory [8, 9].

Since we are working with an external dreibein which is not necessary flat, our results hold for a curved manifold, as long as its topology remains that of flat $\mathcal{R}^{3}$, with asymptotically vanishing curvature. It is the latter two restrictions which allow us to use the general 
results of renormalization theory, established in flat space. Indeed, we may then expand in the powers of the difference between the curved and flat space dreibeins, this difference being considered as an external field in flat space, fastly decreasing asymptotically.

The paper is organized as follows. The Chern-Simons theory coupled to matter, in a curved three-dimensional Riemannian manifold described in terms of external dreibein and spin connection fields, is introduced in Section 2, together with its symmetries. The renormalizability of the model is sketched in Section 3, and our argument leading to the vanishing of the Chern-Simons $\beta$-function is presented in Section 4 . The application of our techniques to the finiteness of the YMCS theory is presented in section f, followed by our conclusions. The paper is completed with two Appendices: In the App. A we compute the classical trace identity whose quantum extension leads to the local form of the Callan-Symanzik equation. The App. B recalls some properties of the short distance Wilson expansion which are used in the main text.

\section{The Chern-Simons Model Coupled With Matter in Curved Space-Time}

\section{The Classical Action}

The gauge field $A_{\mu}^{a}(x)$ lies in the adjoint representation of the gauge group $G$, a general compact Lie group with Lie algebra

$$
\left[X_{a}, X_{b}\right]=i f_{a b}^{c} X_{c}
$$

The scalar matter fields $\varphi_{i}(x)$ and the spinor matter fields $\Psi_{A}(x)$ are in some representations of $G$, the generators being represented by the matrices $T_{a}^{(\varphi)}$ and $T_{a}^{(\Psi)}$, respectively.

Space-time is a three-dimensional Riemannian manifold $\mathcal{M}$, with coordinates $x^{\mu}, \mu=$ $0,1,2$. It is described by a dreibein field $e_{\mu}^{m}(x)$ and its inverse $e_{m}^{\mu}(x), \mu$ being a world index and $m$ a tangent space index. The spin connection $\omega_{\mu}^{m n}(x)$ is not an independent field, but depend on the dreibein due to the vanishing torsion condition. The metric tensor and its inverse read

$$
g_{\mu \nu}(x)=\eta_{m n} e_{\mu}^{m}(x) e_{\nu}^{n}(x), \quad g^{\mu \nu}(x)=\eta^{m n} e_{m}^{\mu}(x) e_{n}^{\nu}(x)
$$

$\eta_{m n}$ being the tangent space flat metric. We denote by $e$ the determinant of $e_{\mu}^{m}$.

As explained in the Introduction, we assume the manifold $\mathcal{M}$ to be topologically equivalent to $\mathcal{R}^{3}$ and asymptotically flat.

The invariances of the theory are:

1) Gauge invariance. The infinitesimal gauge tranformations read - anticipating, we write them as BRS transformations, i.e. we replace their infinitesimal parameters by the anticommuting Faddeev-Popov ghost fields $c^{a}(x)$; we also introduce the antighost fields 
$\bar{c}^{a}(x)$ and the Lagrange multiplyier fields $b^{a}(x)$ which will be used later on in order to define the gauge fixing condition -

$$
\begin{array}{ll}
s A_{\mu}^{a}=-D_{\mu} c^{a} \equiv-\left(\partial_{\mu} c^{a}+f_{b c}{ }^{a} A_{\mu}^{b} c^{c}\right), & s c^{a}=\frac{1}{2} f_{b c}{ }^{a} c^{b} c^{c}, \\
s \Psi_{A}=i c^{a} T_{a}^{(\Psi)}{ }_{A}^{B} \Psi_{B}, & s \bar{c}^{a}=b^{a}, \\
s \varphi_{i}=i c^{a} T_{a}^{(\varphi)}{ }_{i}^{j} \varphi_{j}, & s b^{a}=0 .
\end{array}
$$

The variation of the ghost $c$ is chosen such as to make the BRS operator $s$ nilpotent:

$$
s^{2}=0 \text {. }
$$

2) Invariance under diffeomorphisms. The infinitesimal diffeomorphisms read

$$
\begin{gathered}
\delta_{\text {diff }}^{(\varepsilon)} F_{\mu}=\mathcal{L}_{\varepsilon} F_{\mu}=\varepsilon^{\lambda} \partial_{\lambda} F_{\mu}+\left(\partial_{\mu} \varepsilon^{\lambda}\right) F_{\lambda}, \quad F_{\mu}=A_{\mu}^{a}, e_{\mu}^{m}, \\
\delta_{\text {diff }}^{(\varepsilon)} \Phi=\mathcal{L}_{\varepsilon} \Phi=\varepsilon^{\lambda} \partial_{\lambda} \Phi, \quad \Phi=\varphi_{i}, \Psi_{A}, \quad b^{a}, c^{a}, \bar{c}^{a},
\end{gathered}
$$

where $\mathcal{L}_{\varepsilon}$ is the Lie derivative along the vector field $\varepsilon^{\mu}(x)$ - the infinitesimal parameter of the transformation.

3) Local Lorentz invariance. The infinitesimal transformations, with infinitesimal parameters $\lambda_{[m n]}$, are given by

$$
\delta_{\text {Lorentz }}^{(\lambda)} \Phi=\frac{1}{2} \lambda_{m n} \Omega^{m n} \Phi, \quad \Phi=\text { any field },
$$

with $\Omega^{[m n]}$ acting on $\Phi$ as an infinitesimal Lorentz matrix in the appropriate representation.

The most general classical, power-counting renormalizable action invariant under the diffeomorphisms and local Lorentz transformations, and gauge invariant - i.e. BRSinvariant and independent of the ghost fields - is of the form

$$
\begin{aligned}
\Sigma_{\mathrm{inv}}=\int d^{3} x( & \kappa \varepsilon^{\mu \nu \rho}\left(A_{\mu}^{a} \partial_{\nu} A_{\rho}^{a}+\frac{1}{3} f_{a b c} A_{\mu}^{a} A_{\nu}^{b} A_{\rho}^{c}\right) \\
& \left.+e\left(i \bar{\Psi}_{A} \gamma^{\mu} \mathcal{D}_{\mu} \Psi_{A}+\frac{1}{2} \mathcal{D}_{\mu} \varphi_{i} \mathcal{D}^{\mu} \varphi_{i}-\mathcal{V}(\varphi, \Psi)\right)\right)
\end{aligned}
$$

with the generalized covariant derivative defined by

$$
\mathcal{D}_{\mu} \Phi(x) \equiv\left(\partial_{\mu}-i A_{\mu}^{a}(x) T_{a}^{(\Phi)}+\frac{1}{2} \omega_{\mu}^{m n}(x) \Omega_{m n}\right) \Phi(x)
$$

The function $\mathcal{V}(\varphi, \Psi)$ defines the selfinteractions of the matter fields and their masses:

$$
\mathcal{V}(\varphi, \Psi)=\frac{1}{6} \lambda \varphi^{6}+\frac{1}{2} y \bar{\Psi} \Psi \varphi^{2}+\text { mass terms }+ \text { dimensionful couplings },
$$

whith the short-hand notation

$$
\begin{aligned}
& \lambda \varphi^{6}=\lambda_{i j k l m n} \varphi_{i} \varphi_{j} \varphi_{k} \varphi_{l} \varphi_{m} \varphi_{n}, \\
& y \bar{\Psi} \Psi \varphi^{2}=y_{A B m n} \bar{\Psi}_{A} \Psi_{B} \varphi_{m} \varphi_{n}
\end{aligned}
$$

$\lambda$ and $y$ being invariant tensors of the gauge group. We do not write explicitly the mass terms and the dimensionful couplings since we are interested in the scaling properties in the high energy-momentum limit. 


\section{Gauge Fixing}

The gauge fixing is of the Landau type. It is implemented by adding to the gauge invariant action (2.5) the term

$$
\Sigma_{\mathrm{gf}}=-s \int d^{3} x \operatorname{eg} g^{\mu \nu} \partial_{\mu} \bar{c}_{a} A_{\nu}^{a}=-\int d^{3} x e g^{\mu \nu}\left(\partial_{\mu} b_{a} A_{\nu}^{a}+\partial_{\mu} \bar{c}_{a} D_{\nu} c^{a}\right)
$$

which is BRS invariant due to the nilpotency of $s$.

Moreover, because of the nonlinearity of some of the BRS transformations (2.2), we have also to add a term giving their coupling with external fields, the "antifields" $A_{a}^{* \mu}, c_{a}^{*}$, $\Psi_{A}^{*}, \varphi_{i}^{*}$ :

$$
\Sigma_{\text {ext }}=\int d^{3} x \sum_{\Phi=A_{\mu}^{a}, c^{a}, \Psi_{A}, \varphi_{i}} \Phi^{*} s \Phi .
$$

The antifields are tensorial densities: they transform under the diffeomorphisms as

$$
\begin{aligned}
& \delta_{\text {diff }}^{(\varepsilon)} A_{a}^{* \mu}=\mathcal{L}_{\varepsilon} A_{a}^{* \mu}=\partial_{\lambda}\left(\varepsilon^{\lambda} A_{a}^{* \mu}\right)-\left(\partial_{\lambda} \varepsilon^{\mu}\right) A_{a}^{* \lambda} \\
& \delta_{\text {diff }}^{(\varepsilon)} \Phi^{*}=\mathcal{L}_{\varepsilon} \Phi^{*}=\partial_{\lambda}\left(\varepsilon^{\lambda} \Phi^{*}\right), \quad \Phi^{*}=c_{a}^{*}, \varphi_{i}^{*}, \Psi_{A}^{*} .
\end{aligned}
$$

Their transformations under local Lorentz symmetry are obvious. They are moreover BRS invariant.

From now on we consider the total action

$$
\Sigma=\Sigma_{\mathrm{inv}}+\Sigma_{\mathrm{gf}}+\Sigma_{\mathrm{ext}}
$$

\section{The Functional Identities}

The various symmetries of the model as well as the gauge fixing we have choosen may be expressed as functional identities obeyed by the classical action (2.10).

The BRS invariance is expressed through the Slavnov-Taylor identity

$$
\mathcal{S}(\Sigma)=\int d^{3} x \sum_{\Phi=A_{\mu}^{a}, c^{a}, \Psi_{A}, \varphi_{i}} \frac{\delta \Sigma}{\delta \Phi^{*}} \frac{\delta \Sigma}{\delta \Phi}+b \Sigma=0, \quad \text { with } b=\int d^{3} x b^{a} \frac{\delta}{\delta \bar{c}^{a}} .
$$

For later use we introduce the linearized Slavnov-Taylor operator

$$
\mathcal{B}_{\Sigma}=\int d^{3} x \sum_{\Phi=A_{\mu}^{a}, c^{a}, \Psi_{A}, \varphi_{i}}\left(\frac{\delta \Sigma}{\delta \Phi^{*}} \frac{\delta}{\delta \Phi}+\frac{\delta \Sigma}{\delta \Phi} \frac{\delta}{\delta \Phi^{*}}\right)+b
$$

$\mathcal{S}$ and $\mathcal{B}$ obey the algebraic identity

$$
\mathcal{B}_{\mathcal{F}} \mathcal{B}_{\mathcal{F}} \mathcal{F}^{\prime}+\left(\mathcal{B}_{\mathcal{F}^{\prime}}-b\right) \mathcal{S}(\mathcal{F})=0
$$

$\mathcal{F}$ and $\mathcal{F}^{\prime}$ denoting arbitrary functionals of ghost number zero. From the latter follow

$$
\mathcal{B}_{\mathcal{F}} \mathcal{S}(\mathcal{F})=0 \quad, \quad \forall \mathcal{F}
$$




$$
\left(\mathcal{B}_{\mathcal{F}}\right)^{2}=0 \text { if } \mathcal{S}(\mathcal{F})=0
$$

In particular, since the action $\Sigma$ obeys the Slavnov-Taylor identity, we have the nilpotency property (2.11):

$$
\left(\mathcal{B}_{\Sigma}\right)^{2}=0
$$

In addition to the Slavnov-Taylor identity (2.11), the action (2.10) satisfies the following constraints:

- the Landau gauge condition:

$$
\frac{\delta \Sigma}{\delta b_{a}}=\partial_{\mu}\left(e g^{\mu \nu} A_{\nu}^{a}\right)
$$

- the "antighost equation", peculiar to the Landau gauge [21,

$$
\overline{\mathcal{G}}^{a} \Sigma=\int d^{3} x\left(\frac{\delta}{\delta c^{a}}+f^{a b c} \bar{c}_{b} \frac{\delta}{\delta b^{c}}\right) \Sigma=\Delta_{\mathrm{cl}}^{a}
$$

with

$$
\Delta_{\mathrm{cl}}^{a}=\int d^{3} x\left(f^{a b c}\left(A_{b}^{* \mu} A_{c \mu}-c_{b}^{*} c_{c}\right)+i \bar{\Psi}^{*} T_{a}^{(\Psi)} \Psi-i \varphi^{*} T_{a}^{(\varphi)} \varphi\right),
$$

(The right-hand side of (2.18) being linear in the quantum fields will not get renormalized.)

- the Ward identities for the invariances under the diffeomorphisms (2.3) and the local Lorentz transformations (2.4):

$$
\mathcal{W}_{\text {diff }} \Sigma=\int d^{3} x \sum_{\Phi} \delta_{\text {diff }}^{(\varepsilon)} \Phi \frac{\delta \Sigma}{\delta \Phi}=0
$$

and

$$
\mathcal{W}_{\text {Lorentz }} \Sigma=\int d^{3} x \sum_{\Phi} \delta_{\text {Lorentz }}^{(\lambda)} \Phi \frac{\delta \Sigma}{\delta \Phi}=0
$$

where the summations run over all quantum and external fields.

The functional operators defined here above, together with the operators

$$
\begin{aligned}
& \mathcal{G}_{a}=\frac{\delta}{\delta \bar{c}_{a}}+\partial_{\mu}\left(e g^{\mu \nu} \frac{\delta}{\delta A_{a}^{* \nu}}\right), \\
& \mathcal{W}_{\text {rigid }}^{a}=\int d^{3} x\left(\sum_{\phi=A, c, \bar{c}, b, A^{*}, c^{*}} f^{a b c} \phi_{b} \frac{\delta}{\delta \phi^{c}}+\sum_{\Phi=\Psi, \varphi, \Psi^{*}, \varphi^{*}} T^{(\Phi) a} \Phi \frac{\delta}{\delta \Phi}\right) .
\end{aligned}
$$

obey the algebra (2.13), (2.14) and

$$
\begin{gathered}
\frac{\delta \mathcal{S}(\mathcal{F})}{\delta b_{a}}-\mathcal{B}_{\mathcal{F}}\left(\frac{\delta \mathcal{F}}{\delta b_{a}}-\partial_{\mu}\left(e g^{\mu \nu} A_{\nu}^{a}\right)\right)=\mathcal{G}^{a} \mathcal{F}, \\
\mathcal{G}^{a} \mathcal{S}(\mathcal{F})+\mathcal{B}_{\mathcal{F}} \mathcal{G}^{a} \mathcal{F}=0, \\
\overline{\mathcal{G}}^{a} \mathcal{S}(\mathcal{F})+\mathcal{B}_{\mathcal{F}}\left(\overline{\mathcal{G}}^{a} \mathcal{F}-\Delta_{\text {cl }}^{a}\right)=\mathcal{W}_{\text {rigid }}^{a} \mathcal{F}, \\
\mathcal{W}_{X} \mathcal{S}(\mathcal{F})-\mathcal{B}_{\mathcal{F}} \mathcal{W}_{X} \mathcal{F}=0, \quad X=\text { diff, Lorentz, rigid },
\end{gathered}
$$


where $\mathcal{F}$ is an arbitrary functional of ghost number zero.

One notes that, since the action $\Sigma(2.10)$ obeys the Slavnov-Taylor identity (2.11) and the gauge condition (2.17), the identities (2.22) and (2.24) imply:

- the ghost equation of motion

$$
\mathcal{G}_{a} \Sigma=0,
$$

- the Ward identity expressing the rigid invariance of the theory, i.e. its invariance under the gauge transformations with constant parameters,

$$
\mathcal{W}_{\text {rigid }}^{a} \Sigma=0 \text {. }
$$

The ghost equation (2.26) implies that the theory depends on the field $\bar{c}$ and on the antifield $A^{* \mu}$ through the combination

$$
\hat{A}_{a}^{* \mu}=A_{a}^{* \mu}+e g^{\mu \nu} \partial_{\nu} \bar{c}_{a} .
$$

\section{Renormalizability}

We face now the problem of showing that all the constraints defining the classical theory also hold at the quantum level, i.e., that we can construct a renormalized vertex functional

$$
\Gamma=\Sigma+\mathcal{O}(\hbar)
$$

obeying the same constraints and coinciding with the classical action at order zero in $\hbar$.

As announced in the Introduction, the proof of renormalizability will be valid for manifolds which are topologically equivalent to a flat manifold and which admit an asymptotically flat metric. It is only in this case that one can expand in powers of $\bar{e}_{\mu}^{m}=e_{\mu}^{m}-$ $\delta_{\mu}^{m}$, considering $\bar{e}_{\mu}^{m}$ as a classical background field in flat $\mathcal{R}^{3}$, and thus make use of the general theorems of renormalization theory actually proved for flat space-time [22, 23].

\section{Power-Counting}

The first point to be checked is power-counting renormalizability. It follows from the fact that the dimension of the action is bounded by threef. The ultraviolet dimension as well as the ghost number and the Grassmann parity of all fields and antifields are collected in Table [1.

\footnotetext{
${ }^{5}$ The ultraviolet dimension of the fields coincide with their canonical dimensions. They determine the ultraviolet power-counting. If there are massless fields in the theory, one should take special care of the infrared convergence [24]. We shall not insist on this, since we are interested here in the purely ultraviolet problem of the nonrenormalization of the Chern-Simons coupling. We shall simply assume that no "infrared anomaly" or "radiative mass generation" [25] occurs.
} 


\begin{tabular}{|c||c|c|c|c|c|c|c|c|c|c|c|}
\hline & $A_{\mu}$ & $\Psi$ & $\varphi$ & $b$ & $c$ & $\bar{c}$ & $A^{* \mu}$ & $\Psi^{*}$ & $\varphi^{*}$ & $c^{*}$ & $e_{\mu}{ }^{a}$ \\
\hline \hline$d$ & 1 & 1 & $1 / 2$ & 1 & 0 & 1 & 2 & 2 & $5 / 2$ & 3 & 0 \\
\hline$\Phi \Pi$ & 0 & 0 & 0 & 0 & 1 & -1 & -1 & -1 & -1 & -2 & 0 \\
\hline$G P$ & 0 & 1 & 0 & 0 & 1 & 1 & 1 & 0 & 1 & 0 & 0 \\
\hline
\end{tabular}

Table 1: Ultraviolet dimension $d$, ghost number $\Phi \Pi$ and Grassmann parity GP.

\section{Renormalized Functional Identities}

The second point to be discussed is that of the functional identities which have to be obeyed by the vertex functional.

The gauge condition (2.17), ghost equation (2.26), antighost equation (2.18) as well as rigid gauge invariance (2.27) can easily be shown to hold at all orders, i.e., are not anomalous [20]. The validity to all orders of the Ward identities of diffeomorphisms and local Lorentz will be assumed in the following: the absence of anomalies for them has been proved in refs. [26, 28] for the class of manifolds we are considering here. Therefore we shall be working in the space of diffeomorphism and local Lorentz invariant functionals.

It remains now to show the possibility of implementing the Slavnov-Taylor identity (2.11) for the vertex functional $\Gamma$. As it is well known [20], this amounts to study the cohomology of the nilpotent operator $\mathcal{B}_{\Sigma}$, defined by (2.12), in the space of the local integral functionals $\Delta$ of the various fields involved in the theory. This means that we have to look for solutions of the form

$$
\Delta=\Delta_{\text {cohom }}+\mathcal{B}_{\Sigma} \hat{\Delta}
$$

for the equation

$$
\mathcal{B}_{\Sigma} \Delta=0
$$

$\Delta_{\text {cohom }}$ represents the cohomology, i.e. the "nontrivial" part of the general solution: it cannot be written as a $\mathcal{B}_{\Sigma}$-variation of a local integral functional $\hat{\Delta}$.

The solutions $\Delta$ with ghost number 0 represent the arbitrary invariant counterterms one can add to the action at each order of perturbation theory. The nontrivial ones correspond to a renormalization of the physical parameters: coupling constants and masses, whereas the trivial ones correspond to nonphysical field redefinitions.

The nontrivial solutions with ghost number 1 are the possible gauge anomalies.

In both cases, power-counting renormalizability restricts the dimension of the intgrand of $\Delta$ to 3. Moreover, the constraints 2.17 - 2.20), (2.26) and (2.27), valid now for the vertex functional $\Gamma$, imply for $\Delta$ the conditions

$$
\begin{array}{ll}
\text { (1) } \frac{\delta}{\delta b_{a}} \Delta=0, & \text { (2) } \int d^{3} x \frac{\delta}{\delta c^{a}} \Delta=0, \quad \text { (3) } \mathcal{G}^{a} \Delta=0 \\
\text { (4) } \mathcal{W}_{X} \Delta=0, \quad X=\text { diff., Lorentz, rigid } .
\end{array}
$$

It has been proven in quite generality [27, 28] that in such a gauge theory the coho- 
mology in the sector of ghost number one is independent of the external fields 5 . We can thus restrict the field dependence of $\Delta$ to $A_{\mu}, c, \varphi$, and $\Psi$ - the dependence on $c$ being through its derivatives due to the second of the constraints (3.4).

Beginning with the anomalies (sector of ghost number 1), one knows [26, 28] that, in three dimensions, the cohomology in this sector is empty, up to possible terms in the Abelian ghosts. They however can be seen, by using the arguments of [29], not to contribute to the anomaly, due to the freedom or soft coupling of the Abelian ghosts. We thus conclude to the absence of gauge anomaly, hence to the validity of the Slavnov-Taylor identity (2.11) to all orders for the vertex functional $\Gamma$.

Going now to the sector of ghost number 0, i.e. looking for the arbitrary invariant counterterms which can be freely added to the action at each order, we find that the nontrivial part of $\Delta$ admits the general representation

$$
\Delta_{\text {phys. }}=\left(z_{\kappa} \kappa \frac{\partial}{\partial \kappa}+z_{\lambda} \lambda \frac{\partial}{\partial \lambda}+z_{y} y \frac{\partial}{\partial y}\right) \Sigma,
$$

where we have only kept the terms of dimension 3 (the lower dimension ones having being neglected). We have also neglected terms such as $\int d^{3} x e R \Phi^{2}$, which do not contribute in the limit of flat space. The coefficients $z_{\lambda}$ and $z_{y}$ are invariant tensors, such as the coupling $\lambda$ and $y$ in (2.7).

For the trivial part, we find:

$$
\mathcal{B}_{\Sigma} \hat{\Delta}=\left(z_{A} \mathcal{N}_{A}+z_{\Psi} \mathcal{N}_{\Psi}+z_{\varphi} \mathcal{N}_{\varphi}\right) \Sigma
$$

with

$$
\begin{aligned}
& \mathcal{N}_{A} \Sigma=\left(N_{A}-N_{A^{*}}-N_{b}-N_{\bar{c}}\right) \Sigma=\mathcal{B}_{\Sigma} \int d^{3} x \hat{A}_{a}^{* \mu} A_{\mu}^{a}, \\
& \mathcal{N}_{\Psi} \Sigma=\left(N_{\Psi}+N_{\bar{\Psi}}-N_{\Psi^{*}}-N_{\bar{\Psi}^{*}}\right) \Sigma=-\mathcal{B}_{\Sigma} \int d^{3} x\left(\bar{\Psi}^{* A} \Psi_{A}+\bar{\Psi}^{A} \Psi_{A}^{*}\right), \\
& \mathcal{N}_{\varphi} \Sigma=\left(N_{\varphi}-N_{\varphi^{*}}\right) \Sigma=\mathcal{B}_{\Sigma} \int d^{3} x \varphi^{* i} \varphi_{i},
\end{aligned}
$$

where we have introduced the counting operators

$$
N_{\Phi}=\int d^{3} x \Phi \frac{\delta}{\delta \Phi}, \quad \Phi=\text { any field }
$$

Eqs. (3.5) and (3.6-3.7) make manifest the physical renormalizations, which affect the physical parameters such as the coupling constants (and the masses), on the one hand, and the nonphysical renormalizations, which correspond to a mere redefinition of the field variables, on the other hand.

This concludes the proof of the renormalizability of the model: all functional identities hold without anomaly and the arbitrary renormalizations only affect the parameters defined by the initial classical theory.

\footnotetext{
${ }^{6}$ The proof is valid for any semi-simple gauge group. But if the gauge group contains Abelian factors, the cohomology in the sector of ghost number one may depend on the external fields 27, 28. However, it can be shown [29] that such terms cannot contribute to the gauge anomaly due to the Abelian ghost fields being free or at most softly coupled.
} 


\section{Callan-Symanzik Equation}

The latter renormalization properties are summarized in the Callan-Symanzik equation, which results from the expansion of the invariant insertion defined by

$$
\mathcal{D} \Gamma=\sum_{\text {all dimensionful parameters } \mu} \mu \frac{\partial \Gamma}{\partial \mu},
$$

in the quantum basis defined by (3.5) and (3.6) $-\Sigma$ being replaced there by $\Gamma$ :

$$
\left(\mathcal{D}+\beta_{\kappa} \partial_{\kappa}+\beta_{y} \partial_{y}+\beta_{\lambda} \partial_{\lambda}-\gamma_{A} \mathcal{N}_{A}-\gamma_{\Psi} \mathcal{N}_{\Psi}-\gamma_{\varphi} \mathcal{N}_{\varphi}\right) \Gamma \sim 0
$$

where $\sim$ means equality up to mass terms and dimensionful couplings, the terms contribuiting which vanish in the flat limit being neglected, too.

The aim of the next and last section is to prove that $\beta_{\kappa}$ is vanishing.

\section{Nonrenormalization of the Chern-Simons Coupling}

In order to get more deeply into the scaling properties of the present theory, we need a local form of the Callan-Symanzik equation. This will allow us to exploit the fact that the integrand of the Chern-Simons term in the action is not gauge invariant, although its integral is. Such a local form of the Callan-Symanzik equation is provided by the "trace identity".

In order to derive the latter, let us first introduce the energy-momentum tensor, defined as the following tensorial quantum insertion obtained as the derivative of the vertex functional with respect to the dreibein:

$$
\Theta_{\nu}{ }^{\mu} \cdot \Gamma=e^{-1} e_{\nu}^{m} \frac{\delta \Gamma}{\delta e_{\mu}^{m}} .
$$

From the diffeomorphism Ward identity (2.19), follows the covariant conservation law of the energy-momentum tensor:

$$
e \nabla_{\mu}\left[\Theta_{\nu}^{\mu}(x) \cdot \Gamma\right]=w_{\nu}(x) \Gamma+\nabla_{\mu} w_{\nu}^{\mu}(x) \Gamma,
$$

where $\nabla_{\mu}$ is the covariant derivative with respect to the diffeomorphisms. The right hand side is an equation of motion, the functional differential operators $w_{\lambda}(x)$ and $w_{\nu}{ }^{\mu}(x)$ being given by (A.2) and (A.3), respectively.

The trace $\Theta_{\mu}^{\mu}(x) \cdot \Gamma$ turns out to be vanishing, up to total derivatives, mass terms and dimensionful couplings, in the classical approximation, due to the field equations, which means that (4.1) is the improved energy-momentum tensor. This is shown in Appendix A where we have indeed derived, for the classical theory, the equation

$$
w(x) \Sigma \sim \partial_{\mu} \Lambda^{\mu}(x)
$$


or, equivalently:

$$
e \Theta_{\mu}{ }^{\mu}(x) \sim w^{\text {trace }}(x) \Sigma+\partial_{\mu} \Lambda^{\mu}(x)
$$

with

$$
\begin{gathered}
w=e_{\mu}{ }^{m} \frac{\delta}{\delta e_{\mu}{ }^{m}}-w^{\text {trace }} \\
w^{\text {trace }}=\frac{1}{2} \varphi \frac{\delta}{\delta \varphi}-\frac{1}{2} \varphi^{*} \frac{\delta}{\delta \varphi^{*}}+\Psi \frac{\delta}{\delta \Psi}+\bar{\Psi} \frac{\delta}{\delta \bar{\Psi}}-\Psi^{*} \frac{\delta}{\delta \Psi^{*}}-\bar{\Psi}^{*} \frac{\delta}{\delta \bar{\Psi}^{*}}+\bar{c}^{a} \frac{\delta}{\delta \bar{c}^{a}}+b^{a} \frac{\delta}{\delta b^{a}},
\end{gathered}
$$

and

$$
\Lambda^{\mu}=e i \bar{\Psi} \gamma^{\mu} \Psi+e \varphi \nabla^{\mu} \varphi-s\left(e g^{\mu \nu} \bar{c} A_{\nu}\right) .
$$

The symbol $\sim$ in (4.3) and (4.4) again means equality up to mass terms and dimensionful couplings.

Let us now look for the quantum version of the trace identity (4.3) or (4.4). We first observe that the following commutation relations hold:

$$
\begin{array}{r}
{\left[\frac{\delta}{\delta b^{a}(y)}, w(x)\right]=-\delta(x-y) \frac{\delta}{\delta b^{a}(x)}, \quad\left[\overline{\mathcal{G}}^{a}, w(x)\right]=0,} \\
{\left[\mathcal{G}^{a}(y), w(x)\right]=-\delta(x-y) \mathcal{G}^{a}(x)+\partial_{\mu} \delta(x-y)\left(e g^{\mu \nu} \frac{\delta}{\delta A_{a}^{* \nu}}\right)(y) .}
\end{array}
$$

Let us then define the quantum extension $\Lambda^{\mu} \cdot \Gamma$ of the classical expression (4.7) as a vector insertion obeying - beyond the conditions of invariance or covariance under $\mathcal{B}_{\Gamma}$, $\mathcal{W}_{\text {diff }}, \mathcal{W}_{\text {Lorentz }}$ and $\mathcal{W}_{\text {rigid }}$ - the constraints

$$
\begin{aligned}
\frac{\delta}{\delta b^{a}(y)}\left[\Lambda^{\mu}(x) \cdot \Gamma\right] & =-\delta(x-y) e g^{\mu \nu} A_{\nu}^{a}, \quad \overline{\mathcal{G}}^{a}\left[\Lambda^{\mu}(x) \cdot \Gamma\right]=0, \\
\mathcal{G}^{a}(y)\left[\Lambda^{\mu}(x) \cdot \Gamma\right] & =\delta(x-y) e g^{\mu \nu} \frac{\delta \Gamma}{\delta A_{a}^{* \nu}},
\end{aligned}
$$

already obeyed in the classical limit. Then the divergence $\partial_{\mu}\left[\Lambda^{\mu} \cdot \Gamma\right]$ will obey the same constraints as $\omega(x) \Gamma$, which read

$$
\begin{aligned}
\frac{\delta}{\delta b^{a}(y)} w(x) \Gamma & =-\partial_{\mu} \delta(x-y)\left(e g^{\mu \nu} A_{\nu}^{a}\right)(y), \quad \overline{\mathcal{G}}^{a} w(x) \Gamma=0, \\
\mathcal{G}^{a}(y) w(x) \Gamma & =\partial_{\mu} \delta(x-y)\left(e g^{\mu \nu} \frac{\delta \Gamma}{\delta A_{a}^{* \nu}}\right)(y),
\end{aligned}
$$

and follow from the commutation relations (4.8).

Thus, the dimension 3 insertion $\Delta \cdot \Gamma$ defined by

$$
w(x) \Gamma \sim \partial_{\mu}\left[\Lambda^{\mu}(x) \cdot \Gamma\right]+\Delta(x) \cdot \Gamma,
$$

- which expresses the quantum corrections to the equation (4.3) - beyond of being invariant or covariant under $\mathcal{B}_{\Gamma}, \mathcal{W}_{\text {diff }}, \mathcal{W}_{\text {Lorentz }}$ and $\mathcal{W}_{\text {rigid }}$, will obey the homogeneous constraints

$$
\frac{\delta}{\delta b^{a}(y)}[\Delta(x) \cdot \Gamma]=0, \quad \overline{\mathcal{G}}^{a}[\Delta(x) \cdot \Gamma]=0, \quad \mathcal{G}^{a}(y)[\Delta(x) \cdot \Gamma]=0 .
$$


We have to expand $\Delta \cdot \Gamma$ in a basis of invariant insertions satisfying the constraints (4.12), keeping only terms of dimension 3. Such a basis is given in the classical approximation by

$$
\left\{\varphi^{6}, \quad \varphi^{2} \bar{\Psi} \Psi, \quad e^{-1} \mathcal{B}_{\Sigma}\left(\hat{A}^{*} A\right), \quad e^{-1} \mathcal{B}_{\Sigma}\left(\bar{\Psi}^{*} \Psi+\bar{\Psi} \Psi^{*}\right), \quad e^{-1} \mathcal{B}_{\Sigma}\left(\varphi^{*} \varphi\right)\right\}
$$

up to total derivative terms which may be reabsorbed in the insertion $\Lambda^{\mu}$.

Note: We have separated out explicitly the cohomologically trivial elements (i.e. $\mathcal{B}_{\Sigma^{-}}$ variation) from the nontrivial ones, and only the terms surviving in the flat limit are kept.

An appropriate quantum extension of this basis is

$$
\left\{e^{-1} n_{\lambda} \cdot \Gamma, \quad e^{-1} n_{y} \cdot \Gamma, e^{-1} n_{A} \cdot \Gamma, e^{-1} n_{\Psi} \cdot \Gamma, e^{-1} n_{\varphi} \cdot \Gamma\right\},
$$

up to total derivative terms which may be reabsorbed in a redefinition of the insertion $\Lambda^{\mu} \cdot \Gamma$. Here, $n_{\lambda}$ and $n_{y}$ are the integrands of the insertions obtained by differentiating the vertex functional with respect to the couplings $\lambda$ and $y$, respectively:

$$
\begin{aligned}
& n_{\lambda} \cdot \Gamma: \quad \int d^{3} x n_{\lambda} \cdot \Gamma=\frac{\partial \Gamma}{\partial \lambda}, \\
& n_{y} \cdot \Gamma: \quad \int d^{3} x n_{y} \cdot \Gamma=\frac{\partial \Gamma}{\partial y} .
\end{aligned}
$$

These integrands - defined up to total derivatives - are choosen such as to satisfy the constraints (4.12).

An obvious choice for $n_{\Psi}$ and $n_{\varphi}$ is:

$$
\begin{aligned}
& n_{\Psi} \cdot \Gamma=-\mathcal{B}_{\Gamma}\left(\bar{\Psi}^{*} \Psi+\bar{\Psi} \Psi^{*}\right)=\bar{\Psi} \frac{\delta \Gamma}{\delta \bar{\Psi}}-\bar{\Psi}^{*} \frac{\delta \Gamma}{\delta \bar{\Psi}^{*}}+\text { conjugate } \\
& n_{\varphi} \cdot \Gamma=\mathcal{B}_{\Gamma}\left(\varphi^{*} \varphi\right)=\varphi \frac{\delta \Gamma}{\delta \varphi}-\varphi^{*} \frac{\delta \Gamma}{\delta \varphi^{*}}
\end{aligned}
$$

Their integrals indeed yield the counting operators $\mathcal{N}_{\Psi}$ and $\mathcal{N}_{\varphi}$ defined by (3.7) where $\Sigma$ is replaced by $\Gamma$. Less obvious is the definition of $n_{A}$, due to the fact that $\hat{A}^{*} A$ is not linear in the quantum fields (see (2.28)). In order to define it properly, keeping both properties satisfied by its classical counterpart, i.e. its BRS invariance and the fact that its integral reproduce the counting operator $\mathcal{N}_{A}$, we use a point splitting regularization $\left(x^{\mu} \longrightarrow x^{\mu} \pm \epsilon^{\mu}\right)$ :

$$
n_{A} \cdot \Gamma=\mathcal{B}_{\Gamma}\left(m_{A} \cdot \Gamma\right)=\mathcal{B}_{\Gamma}\left\{\lim _{\epsilon \rightarrow 0} \hat{A}^{*}(x+\epsilon) A(x-\epsilon)\right\} .
$$

That this limit exists is shown in Appendix B.

Thus, the expansion of $\Delta \cdot \Gamma$ in the basis (4.14) we have just constructed yields, after substitution into (4.11) and use of (4.5), the local trace identity in curved space-time:

$$
\begin{aligned}
& e \Theta_{\mu}^{\mu}(x) \cdot \Gamma \sim \\
& \quad\left\{\beta_{\lambda} \cdot n_{\lambda}+\beta_{y} n_{y}-\gamma_{A} n_{A}-\gamma_{\Psi} n_{\Psi}-\gamma_{\varphi} n_{\varphi}\right\} \cdot \Gamma+w^{\text {trace }}(x) \cdot \Gamma+\partial_{\mu}\left[\Lambda^{\mu}(x) \cdot \Gamma\right]
\end{aligned}
$$

+terms vanishing in the flat limit. 
In order to make the connection between the trace of the energy-momentum tensor and the Callan-Symanzik equation, lets us consider a while the limit of flat space-time, where rigid dilatation symmetry makes sense. In this limit, (4.2) and (4.16) hold with $e=1$ and $\nabla_{\mu}=\partial_{\mu}$. We can therefore define the dilatation current as

$$
\left[\mathcal{D}^{\mu} \cdot \Gamma\right]_{\text {flat }}=x^{\nu}\left[\Theta_{\nu}{ }^{\mu}(x) \cdot \Gamma\right]_{\text {flat }}-x^{\nu}\left[w_{\nu}{ }^{\mu}(x) \cdot \Gamma\right]_{\text {flat }}-\left[\Lambda^{\mu}(x) \cdot \Gamma\right]_{\text {flat }},
$$

which, in the classical approximation, is conserved up to mass terms and dimensionful couplings (see (A.8) ). Thus, for the renormalized theory, in the flat limit, we can write as usually the Callan-Symanzik equation - which is the Ward identity for anomalous dilatation invariance - as the integral trace identity

$$
\int d^{3} x\left[\Theta_{\mu}^{\mu}(x) \cdot \Gamma\right]_{\text {flat }} \sim\left(\beta_{\kappa} \partial_{\kappa}+\beta_{y} \partial_{y}+\beta_{\lambda} \partial_{\lambda}-\gamma_{A} \mathcal{N}_{A}-\gamma_{\Psi} \mathcal{N}_{\Psi}-\gamma_{\varphi} \mathcal{N}_{\varphi}\right) \cdot \Gamma_{\text {flat }} .
$$

This observation allows us to identify the coefficients $\beta$ and $\gamma$ of the expansion (4.16) with those of the Callan-Symanzik equation (3.9). In particular, the absence of a term corresponding to the integrand of the Chern-Simons action in the basis (4.14) of invariant local operators proves the vanishing of the Chern-Simons $\beta$ function:

$$
\beta_{\kappa}=0
$$

\section{The Yang-Mills Chern-Simons Theory in Curved Space-Time}

The YMCS action, invariant under the diffeomorphisms and local Lorentz transformations, and gauge invariant - i.e. BRS-invariant - in the Landau gauge, is of the form

$$
\begin{aligned}
\Sigma_{\mathrm{inv}}+\Sigma_{\mathrm{gf}}= & \int d^{3} x\left\{-\frac{e}{4} F_{\mu \nu}^{a} F^{a \mu \nu}+m \varepsilon^{\mu \nu \rho}\left(A_{\mu}^{a} \partial_{\nu} A_{\rho}^{a}+\frac{g}{3} f_{a b c} A_{\mu}^{a} A_{\nu}^{b} A_{\rho}^{c}\right)\right\} \\
& -\int d^{3} x \operatorname{eg}^{\mu \nu}\left(\partial_{\mu} b_{a} A_{\nu}^{a}+\partial_{\mu} \bar{c}_{a} D_{\nu} c^{a}\right),
\end{aligned}
$$

where $m$ is a dimensionful coupling constant - the topological mass indeed [12] - and $g$ is the gauge coupling constant, dimensionful, too.

The field strength and covariant derivative are defined as:

$$
\begin{gathered}
F_{\mu \nu}^{a}=\partial_{\mu} A_{\nu}^{a}-\partial_{\nu} A_{\mu}^{a}+g f_{a b c} A_{\mu}^{b} A_{\nu}^{c}, \\
D_{\mu} c^{a}=\partial_{\mu} c^{a}+g f_{a b c} A_{\mu}^{b} c^{c} .
\end{gathered}
$$

The BRS transformations and antighost equation now read

$$
\begin{gathered}
s A_{\mu}^{a}=-D_{\mu} c^{a}, \quad s c^{a}=\frac{g}{2} f_{b c}{ }^{a} c^{b} c^{c}, \\
s \bar{c}^{a}=b^{a}, \quad s b^{a}=0 .
\end{gathered}
$$

\footnotetext{
${ }^{7}$ The parametrization of the coupling constant, differing from the one used in the preceding sections, is adapted to the fact that it is now dimensionful.
} 
and

$$
\overline{\mathcal{G}}^{a} \Sigma=\int d^{3} x\left(\frac{\delta}{\delta c^{a}}+g f^{a b c} \bar{c}_{b} \frac{\delta}{\delta b^{c}}\right) \Sigma=\Delta_{\mathrm{cl}}^{a}
$$

with

$$
\overline{\mathcal{G}}^{a}=\int d^{3} x\left(\frac{\delta}{\delta c^{a}}+g f^{a b c} \bar{c}_{b} \frac{\delta}{\delta b^{c}}\right), \quad \Delta_{\mathrm{cl}}^{a}=g \int d^{3} x f^{a b c}\left(A_{b}^{* \mu} A_{c \mu}-c_{b}^{*} c_{c}\right)
$$

The functional identities (2.12), (2.19), (2.20) and the constraints (2.17), (2.26) and (2.27) are unchanged - except for the matter terms which are now absent.

Finally, in order to quantize the system (5.1) we add an action term $\Sigma_{\text {ext }}$ for the coupling of the BRS transformations to external fields:

$$
\Sigma_{\text {ext }}=\int d^{3} x \sum_{\Phi=A_{\mu}^{a}, c^{a}} \Phi^{*} s \Phi .
$$

\section{Renormalizability and Quantum Scale Invariance}

\section{Power-Counting}

The first point to be checked is power-counting renormalizability - in fact superrenormalizability. It follows from the dimension of the action being bounded by three. The ultraviolet dimension, as well as the ghost number and the Grassmann parity of all fields and antifields are collected in Table 2.

\begin{tabular}{|c||c|c|c|c|c|c|c|}
\hline & $A_{\mu}$ & $b$ & $c$ & $\bar{c}$ & $A^{* \mu}$ & $c^{*}$ & $g$ \\
\hline \hline$d$ & $1 / 2$ & $3 / 2$ & $-1 / 2$ & $3 / 2$ & $5 / 2$ & $7 / 2$ & $1 / 2$ \\
\hline$\Phi \Pi$ & 0 & 0 & 1 & -1 & -1 & -2 & 0 \\
\hline$G P$ & 0 & 0 & 1 & 1 & 1 & 0 & 0 \\
\hline
\end{tabular}

Table 2: Ultraviolet dimension $d$, ghost number $\Phi \Pi$ and Grassmann parity $G P$.

In order to explicitely find the possible renormalizations and anomalies of the theory, we can use the following result [17]: The degree of divergence of a 1-particle irreducible Feynman graph $\gamma$ is given by

$$
d(\gamma)=3-\sum_{\Phi} d_{\Phi} N_{\Phi}-\frac{1}{2} N_{g}
$$

Here $N_{\Phi}$ is the number of external lines of $\gamma$ corresponding to the field $\Phi, d_{\Phi}$ is the dimension of $\Phi$ as given in Table 2 , and $N_{g}$ is the power of the coupling constant $g$ in the integral corresponding to the diagram $\gamma$. The dependence on the coupling constant is characteristic of a superrenormalizable theory.

The equivalent expression

$$
d(\gamma)=4-\sum_{\Phi}\left(d_{\Phi}+\frac{1}{2}\right) N_{\Phi}-L
$$


where $L$ is the number of loops of the diagram, shows that only graphs up to two-loop order are divergent.

In order to apply the known results on the quantum action principle 23] to the present situation, one may consider $g$ as an external field of dimension $\frac{1}{2}$. Including it in the summation under $\Phi$, (6.1) gets the same form as in a strictly renormalizable theory:

$$
d(\gamma)=3-\sum_{\tilde{\Phi}=\Phi, g} d_{\tilde{\Phi}} N_{\tilde{\Phi}}, \quad \text { with } \quad d_{g}=\frac{1}{2} .
$$

Thus, including the dimension of $g$ into the calculation, we may state that the dimension of the counterterms of the action is bounded by 3. But, since they are generated by loop graphs, they are of order 2 in $g$ at least. This means that, not taking now into account the dimension of $g$, we can conclude that their real dimension is bounded by 2 . The same holds for the possible breakings of the Slavnov-Taylor identity.

The absence of anomaly for the Slavnov-Taylor identity holds here in the same way as previously discussed, in Sect.3.

Let us now look for the arbitrary invariant counterterms which can be freely added to the action at each order. According to the above discussion the counterterm is at least of order $g^{2}$. Thus, the most general expression for the nontrivial part of $\Delta$ reads

$$
\Delta_{\text {phys. }}=z_{m} \int d^{3} x m \varepsilon^{\mu \nu \rho}\left(A_{\mu}^{a} \partial_{\nu} A_{\rho}^{a}+\frac{g}{3} f_{a b c} A_{\mu}^{a} A_{\nu}^{b} A_{\rho}^{c}\right),
$$

where $z_{m}$ is arbitrary parameter. Expression (6.4) admits the suitable representation

$$
\Delta_{\text {phys. }}=z_{m} m \frac{\partial}{\partial m} \Sigma,
$$

where $\Sigma$ is the classical action (2.10). Eq. (6.4) shows that a priori only the parameter $m$ can get radiative corrections. This means that the $\beta$-function related to the gauge coupling constant $g$ is vanishing to all orders of perturbation theory, and the anomalous dimensions of the fields as well. We can just state that the radiative corrections can be reabsorbed through a redefinition of the topological mass only. This concludes the proof of the renormalizability of the theory: all functional identities hold without anomaly and the renormalizations might only affect the the Chern-Simons coupling, i.e. the topological mass $m$. But the latter turns out to be not renormalized, too. We shall indeed show in the next Section that the corresponding $\beta$-function vanishes.

\section{Quantum Scale Invariance}

The argument is very similar to the one presented in Sect. 4. However, the equations 4.3 - 4.7) for the classical theory are now replaced by

$$
w(x) \Sigma \equiv\left(e_{\mu}^{a}(x) \frac{\delta}{\delta e_{\mu}^{a}(x)}-w^{\text {trace }}(x)\right) \Sigma=\Lambda(x),
$$

\footnotetext{
${ }^{8}$ See appendix A.
} 
or, equivalently:

$$
e \Theta_{\mu}{ }^{\mu}(x)=w^{\text {trace }}(x) \Sigma+\Lambda(x)
$$

with

$$
w^{\text {trace }}(x)=-\frac{1}{2}\left(A_{\mu}^{a} \frac{\delta}{\delta A_{\mu}^{a}}-A_{a}^{* \mu} \frac{\delta}{\delta A_{a}^{* \mu}}+c^{a} \frac{\delta}{\delta c^{a}}-c_{a}^{*} \frac{\delta}{\delta c_{a}^{*}}\right)+\frac{3}{2}\left(\bar{c}^{a} \frac{\delta}{\delta \bar{c}^{a}}+b^{a} \frac{\delta}{\delta b^{a}}\right)
$$

and

$\Lambda=\frac{m}{2} \varepsilon^{\mu \nu \lambda} A_{\mu}^{a} F_{\nu \lambda}^{a}-\frac{g}{2} f_{a b c}\left(e F^{\mu \nu a} A_{\mu}^{b} A_{\nu}^{c}+\hat{A}^{* \mu a} A_{\mu}^{b} c^{c}-\frac{1}{2} c^{* a} c^{b} c^{c}\right)+$ total derivative terms,

$\Lambda$ being invariant under $\mathcal{B}_{\Sigma}$.

Note: The latter is the effect of the breaking of scale invariance due to the dimensionful couplings. The dimension of $\Lambda$ - the dimensions of $g$ and $m$ not being taken into account - is lower than three: it is a soft breaking.

The commutation relations (4.8) are changed accordingly into:

$$
\begin{aligned}
{\left[\frac{\delta}{\delta b^{a}(y)}, w(x)\right] } & =-\frac{3}{2} \delta(x-y) \frac{\delta}{\delta b^{a}(x)} \\
{\left[\mathcal{G}^{a}(y), w(x)\right] } & =-\frac{3}{2} \delta(x-y) \mathcal{G}^{a}(x)+\frac{3}{2} \partial_{\mu} \delta(x-y)\left(e g^{\mu \nu} \frac{\delta}{\delta A_{a}^{* \nu}}\right)(y), \\
{\left[\overline{\mathcal{G}}^{a}, w(x)\right] } & =\frac{1}{2} \frac{\delta}{\delta c^{a}(x)} .
\end{aligned}
$$

Now the relations (6.10) applied to the vertex functional $\Gamma$ yield for insertion $w(x) \Gamma$ the properties

$$
\begin{aligned}
\frac{\delta}{\delta b_{a}(y)} w(x) \Gamma & =-\frac{3}{2} \partial_{\mu} \delta(x-y)\left(e g^{\mu \nu} A_{\nu}^{a}\right)(y), \\
\mathcal{G}^{a}(y) w(x) \Gamma & =\frac{3}{2} \partial_{\mu} \delta(x-y)\left(e g^{\mu \nu} \frac{\delta \Gamma}{\delta A_{a}^{* \nu}}\right)(y), \\
\overline{\mathcal{G}}^{a} w(x) \Gamma & =\frac{1}{2} \frac{\delta \Gamma}{\delta c_{a}(x)} .
\end{aligned}
$$

where we again use the fact that the constraints (2.17), (2.18) and (2.26) can be maintained at the quantum level.

The quantum version of (6.6) or (6.7) will be written as

$$
w(x) \Gamma=\Lambda(x) \cdot \Gamma+\Delta(x) \cdot \Gamma,
$$

where $\Lambda(x) \cdot \Gamma$ is some quantum extension of the classical insertion (6.9), subjected to the same constraints (6.11) as $w(x) \Gamma$. It follows that the insertion $\Delta \cdot \Gamma$ defined by (6.12) 
obeys the homogeneous constraints (4.12), beyond of the usual invariances or covariances. The power-counting dimension of $\Delta$ is 3 , but being an effect of the radiative corrections, it possess a factor $g^{2}$ at least, and thus its effective dimension is at most two - let us recall that we have attributed power-counting dimension $1 / 2$ to $g$. It turns out that there is no insertion obeying all these constraints - the Yang-Mills Lagrangian density has terms of dimension 3 (without factor $g^{2}$ ) and the Chern-Simons Lagrangian density is not BRS invariant. Hence $\Delta \cdot \Gamma=0$ : there is no radiative correction to the insertion $\Lambda \cdot \Gamma$ describing the breaking of scale invariance, and (6.12) becomes

$$
e \Theta_{\mu}^{\mu}(x) \cdot \Gamma=w^{\text {trace }}(x)+\Lambda(x) \cdot \Gamma \text {. }
$$

As in Sect. ॠ, this local trace identity leads to a Callan-Symanzik equation:

$$
\mathcal{D} \Gamma=\int d^{3} x \Lambda(x) \cdot \Gamma
$$

but now with no radiative effect at all: the $\beta$-functions associated to the parameters $g$ and $m$ both vanish, scale invariance remaining affected only by the soft breaking $\Lambda$.

\section{Conclusions}

We have thus achieved the proof of absence of renormalization of the Chern-Simons coupling (Eq. (4.19)) for the Chern-Simons theory coupled with matter through a direct use of the nongauge invariance of the Chern-Simons three-form. As a by-product, we have obtained a trace identity valid in curved space-time (Eq. (4.16)), equivalent to a local version of the Callan-Symanzik equation (3.9), the result (4.19) being taken into account. The same techniques has allowed us to give a simple proof of the finiteness of the Yang-Mills - Chern-Simons theory.

\section{Acknowledgements}

We thank Sylvain Wolf for a useful critical reading of the manuscript, and the authors of Refs. 18, 19 for interesting discussions on the subject of the present paper and on their work. This work has been done in parts at the CBPF-DCP, at the Dept. of Physics of the UFMG and at the the Dept. of Physics of the UFES. We thank all these three institutions for their financial help for travel expenses and for their kind hospitality. One of the authors (O.M.D.C.) dedicates this work to his wife, Zilda Cristina, to his daughter, Vittoria, and to his son, Enzo, who is coming. 


\section{Appendix A: Trace Identity and Dilatation Invariance for the Classical Theory}

\section{Chern-Simons Theory coupled to Matter:}

The conservation of the energy-momentum tensor $\Theta_{\lambda}{ }^{\mu}$ is a consequence of the diffeomorphism Ward Identity (2.19) and of the definition (4.1), yielding the following local equation

$$
e \nabla_{\mu} \Theta_{\lambda}^{\mu}(x)=w_{\lambda}(x) \Sigma+\nabla_{\mu} w_{\lambda}^{\mu}(x) \Sigma,
$$

where $\nabla_{\mu}$ is the covariant derivative with respect to the diffeomorphisms w with the differential operators $w_{\lambda}(x)$ and $w_{\lambda}^{\mu}(x)$ acting on $\Sigma$ representing contact terms:

$$
w_{\lambda}(x)=\sum_{\text {all fields }}\left(\nabla_{\lambda} \Phi\right) \frac{\delta}{\delta \Phi},
$$

(becoming the translation Ward operator in the limit of flat space), and

$$
\begin{aligned}
& w_{\lambda}^{\mu}(x)= \\
& A^{* \mu} \frac{\delta}{\delta A^{* \lambda}}-A_{\lambda} \frac{\delta}{\delta A_{\mu}}-\delta_{\lambda}{ }^{\mu}\left[\varphi^{*} \frac{\delta}{\delta \varphi^{*}}+\Psi^{*} \frac{\delta}{\delta \Psi^{*}}+\bar{\Psi}^{*} \frac{\delta}{\delta \bar{\Psi}^{*}}+c^{*} \frac{\delta}{\delta c^{*}}+A^{* \mu} \frac{\delta}{\delta A^{* \mu}}\right] .
\end{aligned}
$$

The integral of the trace of the tensor $\Theta_{\lambda}{ }^{\mu}$,

$$
\int d^{3} x e \Theta_{\mu}^{\mu}=\int d^{3} x e_{\mu}^{m} \frac{\delta \Sigma}{\delta e_{\mu}{ }^{m}} \equiv N_{e} \Sigma,
$$

turns out to be an equation of motion - which means that $\Theta_{\lambda}{ }^{\mu}$ is the improved energymomentum tensor. This follows from the identity, which is easily checked by inspection of the classical action

$$
N_{e} \Sigma \sim\left(N_{\Psi}+N_{\bar{\Psi}}+\frac{1}{2} N_{\varphi}+N_{b}+N_{\bar{c}}-N_{\Psi^{*}}-N_{\bar{\Psi}^{*}}-\frac{1}{2} N_{\varphi^{*}}\right) \Sigma,
$$

where $\sim$ means equality up to mass terms and dimensionful couplings, and the $N_{\Phi}$ 's are the counting operators defined by (3.8). $N_{e}$ is the counting operator of the dreibein field $e_{\mu}^{m}$. It is interesting to note that (A.4) is nothing but the Ward identity for rigid Weyl symmetry 30 - broken by the mass terms and dimensionful couplings.

${ }^{9}$ For a tensor $T$, such as, e.g., $A_{\mu}$ or $\delta / \delta A^{* \mu}$ :

$$
\nabla_{\lambda} T_{\nu \cdots}^{\mu \cdots}=\partial_{\lambda} T_{\nu \cdots}^{\mu \cdots}+\Gamma_{\lambda}^{\mu}{ }_{\rho} T_{\nu \cdots}^{\rho \cdots}+\cdots-\Gamma_{\lambda}{ }^{\rho}{ }_{\nu} T_{\rho \cdots}^{\mu \cdots}-\cdots,
$$

where the $\Gamma$. 's are the Christoffel symbols corresponding to the connexion $\omega_{\mu}^{m n}$. The covariant derivative of a tensorial density $\mathcal{T}$, such as, e.g., $A^{* \mu}$ or $\delta / \delta A_{\mu}$, is related to that of the tensor $e^{-1} \mathcal{T}$ by:

$$
\nabla_{\lambda} \mathcal{T}_{\nu \cdots}^{\mu \cdots}=e \nabla_{\lambda}\left(e^{-1} \mathcal{T}_{\nu \cdots}^{\mu \cdots}\right)
$$


Considering the integrand of (A.5), taking (A.4) into account, we may write:

$$
w(x) \Sigma \sim \partial_{\mu} \Lambda^{\mu}(x),
$$

with $w(x)$ and $\Lambda^{\mu}(x)$ given by (4.6- 4.7).

We now come to scale invariance, in the flat limit. The classical dilatation current $\mathcal{D}^{\mu}$ can be defined as

$$
\mathcal{D}^{\mu}(x)=x^{\lambda} \Theta_{\lambda}^{\mu}(x)-x^{\lambda}\left[w_{\lambda}^{\mu}(x) \Sigma\right]-\Lambda^{\mu}(x) .
$$

It obeys, up to mass terms, dimensionful couplings and total derivatives, the conservation Ward identity, a direct consequence of (A.1) and (A.6):

$$
\partial_{\mu} \mathcal{D}^{\mu} \sim w_{D}(x) \Sigma
$$

where

$$
w_{D}(x)=w^{\text {trace }}-w_{\mu}^{\mu}=\sum_{\text {all fields }}\left[\left(d_{\Phi}+x \cdot \partial\right) \Phi\right] \frac{\delta}{\delta \Phi},
$$

is the local operator for the scale transformations and $d_{\Phi}$ is the dimension of the fields surviving in the flat limit (see Table 1).

\section{Yang-Mills Chern-Simons Theory:}

The equations (A.1 - A.3) for the conservation of the energy-momentum tensor are unchanged - except the matter terms which are now absent. The expression for the righthand side of (A.5) is modified into

$$
N_{e} \Sigma=\left(-\frac{1}{2} N_{A}+\frac{1}{2} N_{A^{*}}+\frac{3}{2} N_{b}+\frac{3}{2} N_{\bar{c}}-\frac{1}{2} N_{c}+\frac{1}{2} N_{c^{*}}\right) \Sigma+\left(m \partial_{m}+\frac{1}{2} g \partial_{g}\right) \Sigma .
$$

with now a strict equality sign since we keep the contributions from all terms of the action (5.1).

The correspondent of the local identity (A.6) reads

$$
w(x) \Sigma=\Lambda(x),
$$

with $w$ and $\Lambda$ given by $(6.6-6.9)$

Finally, as in (A.8), we obtain the broken conservation Ward identity

$$
\partial_{\mu} \mathcal{D}^{\mu}=w_{D}(x) \Sigma+\Lambda(x) .
$$

The local dilatation operator $w_{D}(x)$ is given by (A.9), but the dimensions $d_{\Phi}$ given in Table 2 . 


\section{Appendix B: Definition of the Bilinear Insertion (4.15) Through the Wilson-Zimmermann Short Distance Expansion}

The aim is to construct two local insertions $m_{A}(x)$ and $n_{A}(x)$, of ghost number -1 and 0 , respectively, invariant - or covariant - under BRS, the diffeomorphisms and the local lorentz transformations, and such that

$$
n_{A}(x) \cdot \Gamma=\mathcal{B}_{\Gamma}\left[m_{A}(x) \cdot \Gamma\right]
$$

and

$$
\int d^{3} x n_{A}(x) \cdot \Gamma=\mathcal{N}_{A} \Gamma
$$

where $\mathcal{N}_{A}$ is the counting operator defined by (3.7). The classical solution of the problem is given by

$$
\begin{aligned}
& m_{A}(x)=\hat{A}^{* \mu} A_{\mu}, \\
& n_{A}(x)=A_{\mu}\left(\frac{\delta \Sigma}{\delta A_{\mu}}+e g^{\mu \nu} \partial_{\nu} b\right)-\hat{A}^{* \mu} \frac{\delta \Sigma}{\delta \hat{A}^{* \mu}},
\end{aligned}
$$

with $\hat{A}^{* \mu}$ defined by 2.28 ).

In order to find their quantum extensions, let us consider the point splitted insertions introduced in (4.15):

$$
\begin{aligned}
& m_{A}^{e}(x) \cdot \Gamma=\hat{A}^{* \mu}\left(x_{+}\right) A_{\mu}\left(x_{-}\right) \cdot \Gamma, \\
& n_{A}^{e}(x) \cdot \Gamma=\mathcal{B}_{\Gamma}\left[m_{A}^{e}(x) \cdot \Gamma\right],
\end{aligned}
$$

with $x_{ \pm}^{\mu}=x^{\mu} \pm \epsilon^{\mu}$, and let us show that the limit $\epsilon \rightarrow 0$ exists and solves the problem.

The Wilson-Zimmermann short distance expansion [31] yieldst0

$$
m_{A}^{e}(x) \cdot \Gamma=\sum_{K} f_{K}(\epsilon) B_{K}(x) \cdot \Gamma+R^{e}(\epsilon, x)
$$

where $R^{e}$ goes to zero in the limit $\epsilon \rightarrow 0$. The $B_{K}(x)$ 's are all possible renormalized insertions (Zimmermann's normal products [22]) of dimension $\leq 3$ (the dimension of $\left.m_{A}\right)$, and the coefficients $f_{K}(\epsilon)$ have a short distance behaviour

$$
f_{K}(\epsilon) \sim|\epsilon|^{-d_{K}} \quad \text { up to logarithmic factors, } \quad d_{K} \leq 3-\operatorname{dim}\left(B_{K}\right) .
$$

Taking into account all the constraints which must be obeyed by $m_{A}$, we see that the list of possible operators $B_{K}$ reduces to quantum extensions of the three following expressions:

$$
\begin{array}{ll}
B_{1}=\hat{A}^{* \mu} A_{\mu} & d_{1} \leq 0, \\
B_{2}=\bar{\Psi}^{*} \Psi+\bar{\Psi} \Psi^{*} & d_{2} \leq 0, \\
B_{3}=\varphi^{*} \varphi & d_{3} \leq 0 .
\end{array}
$$

\footnotetext{
${ }^{10}$ The short distance expansion has been rigorously proved only for theories without massless fields. The extension to massless fields is assumed to hold.
} 
Now, we observe that the integral of $m_{A}^{e}$ can be written, after partial integration of the term involving the antighost field $\bar{c}$, and use of the gauge condition (2.17), as

$$
\int d^{3} x m_{A}^{e}(x) \cdot \Gamma=\int d^{3} x\left(A^{* \mu}\left(x_{+}\right) A_{\mu}\left(x_{-}\right)-\bar{c}\left(x_{+}\right) \frac{\delta \Gamma}{\delta b\left(x_{-}\right)}\right),
$$

which has the well defined limit

$$
\int d^{3} x\left(A^{* \mu}(x) A_{\mu}(x)-\bar{c}(x) \frac{\delta \Gamma}{\delta b(x)}\right),
$$

for $\epsilon \rightarrow 0$. Since there is no total derivative term in the expansion (B.5), we conclude from this that all three coefficients $f_{K}(\epsilon)$ are regular at $\epsilon=0$. This proves the existence of the limit

$$
m_{A}(x) \cdot \Gamma=\lim _{\epsilon \rightarrow 0} m_{A}^{e}(x) \cdot \Gamma,
$$

with the integral $\int d^{3} x m_{A}(x) \cdot \Gamma$ being equal to the expression (B.7).

The existence of the limit

$$
n_{A}(x) \cdot \Gamma=\lim _{\epsilon \rightarrow 0} n_{A}^{e}(x) \cdot \Gamma
$$

with the properties $(\mathbb{B} .1)$ and (B.2) follows by acting with the BRS operator $\mathcal{B}_{\Gamma}$ on $(\mathbb{B} .8)$.

\section{References}

[1] D. Birmingham, M. Blau, M. Rakowski and G. Thompson, Phys. Rep. 209 (1991) 129;

[2] A.S. Schwarz, Baku International Topological Conference, Abstracts, vol. 2, p. 345 (1987);

[3] E. Witten, Commun. Math. Phys. 117 (1988) 353;

Commun. Math. Phys. 118 (1988)601;

[4] E. Witten, Commun. Math. Phys. 121 (1989) 351;

[5] E. Witten, Nucl. Phys. B311 (1988) 46;

[6] S. Deser, J. McCarthy and Z. Yang, Phys. Lett. B222 (1989) 61;

[7] G. Moore and N. Seiberg, Phys. Lett. B220 (1989) 442;

M. Bos and V.P. Nair, Phys. Lett. B223 (1989) 61;

J.M.F. Labastida and A.V. Ramallo, Phys. Lett. B227 (1989) 92;

S. Emery and O. Piguet, Helv. Phys. Acta 64 (1991) 1256;

[8] E. Guadagnini, M. Martellini and M. Mintchev, Phys. Lett. B227 (1989) 111;

Nucl. Phys. B330 (1990) 575; 
[9] A. Blasi and R. Collina, Nucl. Phys. B345 (1990) 472;

F. Delduc, C. Lucchesi, O. Piguet and S.P. Sorella, Nucl. Phys. B346 (1990) 313;

C. Lucchesi and O. Piguet, Nucl. Phys. B381 (1992) 281;

[10] A. Blasi, N. Maggiore and S.P. Sorella, Phys. Lett. B285 (1992) 54;

[11] G.A.N. Kapustin and P.I. Pronin, Phys. Lett. B318 (1993) 465;

[12] S. Deser, R. Jackiw and S. Templeton, Ann. Phys. 140 (1982) 372 and 185 (1988) 406;

[13] C.P. Martin, Phys. Lett. B241 (1990) 513;

[14] R. Pisarski and S. Rao, Phys. Rev. D32 (1985) 2081;

[15] G. Giavarini, C.P. Martin and F. Ruiz Ruiz, Nucl. Phys. B381 (1992) 222;

[16] F. Ruiz Ruiz and P. van Nieuwenhuizen, Nucl. Phys. B486 (1997) 443;

[17] N. Maggiore, O. Piguet and M. Ribordy, Helv. Phys. Acta 68 (1995) 265;

[18] V.E.R Lemes, C. Linhares de Jesus, C.A.G. Sasaki, S.P. Sorella, L.C.Q. Vilar and O.S. Ventura, "A simple remark on three dimensional gauge theories", preprint CBPF-NF-050/97, hep-th/9708098, to appear in Phys. Lett. B;

[19] V.E.R. Lemes, C. Linhares de Jesus, S.P. Sorella, L.C.Q. Vilar and O.S. Ventura, "Chern-Simons as a geometrical set up for three dimensional gauge theories", preprint CBPF-NF-078/97, hep-th/9801021;

[20] O. Piguet and S.P. Sorella, "Algebraic Renormalization", Lecture Notes in Physics, m28, Springer-Verlag, Berlin, Heidelberg, 1995;

[21] A. Blasi, O. Piguet and S. P. Sorella, Nucl. Phys. B356 (1991) 154;

[22] W. Zimmermann, 1970 Brandeis Lectures, Lectures on Elementary Particle and Quantum Field Theory, eds. S. Deser, M. Grisaru and H. Pendleton (MIT Press Cambridge); Ann. Phys. (N.Y.) 77 (1973) 536;

[23] J.H. Lowenstein, Phys. Rev. D4 (1971) 2281; Commun. Math. Phys. 24 (1971) 1;

Y.M.P. Lam, Phys. Rev. D6 (1972) 2145; Phys. Rev. D7 (1973) 2943;

T.E. Clark and J.H. Lowenstein, Nucl. Phys. B113 (1976) 109;

[24] J.H. Lowenstein, Commun. Math. Phys. 47 (1976) 53; "BPHZ Renormalization" in Renormalization Theory, eds. G. Velo and A.S. Wightman (D. Reidel, Dordrecht, 1976);

[25] S. Weinberg, Phys. Rev. D7 (1973) 2887;

G. Bandelloni, C. Becchi, A. Blasi and R. Collina,

Commun. Math. Phys. 67 (1979) 147;

W. Bardeen, O. Piguet and K. Sibold, Phys. Lett. B72 (1977) 231;

O. Piguet, M. Schweda and K. Sibold, Nucl. Phys. B168 (1980) 337;

[26] F. Brandt, N. Dragon and M. Kreuzer, Nucl. Phys. B340 (1990) 187; 
[27] G. Barnich and M. Henneaux, Phys. Rev. Lett. 72 (1994) 1558;

[28] G. Barnich, F. Brandt and M. Henneaux, Nucl. Phys. B455 (1995) 357;

[29] G. Bandelloni, C. Becchi, A. Blasi and R. Collina, Ann. Inst. Henri Poincaré 28 (1978) 225,255;

[30] A. Iorio, L. O'Raifeartaigh, I. Sachs and C. Wiesendager, Nucl. Phys. B495 (1997) 433;

[31] K.G. Wilson and W. Zimmermann, Commun. Math. Phys. 24 (1972) 87;

W. Zimmermann, Ann. Phys. (N.Y.) 77 (1973) 570; 